\title{
BMJ Open Study protocol for SFX-01 after subarachnoid haemorrhage (SAS): a multicentre randomised double- blinded, placebo controlled trial
}

\author{
Ardalan H Zolnourian (D , ,,2 Stephen Franklin, ${ }^{3}$ Ian Galea, ${ }^{1,4}$ \\ Diederik Oliver Bulters ${ }^{1,2}$
}

To cite: Zolnourian AH, Franklin S, Galea I, et al. Study protocol for SFX-01 after subarachnoid haemorrhage (SAS): a multicentre randomised double-blinded, placebo controlled trial. BMJ Open 2020;10:e028514. doi:10.1136/ bmjopen-2018-028514

- Prepublication history for this paper is available online. To view these files, please visit the journal online (http://dx.doi org/10.1136/bmjopen-2018028514).

IG and DOB are joint senior authors.

Received 13 December 2018 Revised 26 December 2019 Accepted 04 February 2020

Check for updates

(C) Author(s) (or their employer(s)) 2020. Re-use permitted under CC BY-NC. No commercial re-use. See rights and permissions. Published by BMJ.

${ }^{1}$ Department of Clinical Neurosciences, University of Southampton, Southampton, UK ${ }^{2}$ Department of Neurosurgery, University Hospital Southampton NHS Foundation Trust,

Southampton, UK

${ }^{3}$ Evgen Pharma, Liverpool, UK

${ }^{4}$ Department of Experimental

Neurology, Faculty of Medicine, University of Southampton, Southampton, UK

Correspondence to Dr Ardalan H Zolnourian; a.zolnourian@soton.ac.uk

\section{ABSTRACT}

Introduction Subarachnoid haemorrhage (SAH) from a ruptured cerebral aneurysm carries high morbidity and mortality. Despite huge advances in techniques to secure the aneurysm, there has been little progress in the treatment of the deleterious effects of the haemorrhage. Sulforaphane is an Nrf2 inducer with anti-oxidant and antiinflammatory properties. It has been shown to improve clinical outcome in experimental models of SAH, but is unstable. SFX-01 (Evgen Pharma) is a novel composition comprised of synthetic sulforaphane stabilised within an $\alpha$-cyclodextrin complex. On ingestion, the complex releases sulforaphane making SFX-01 an ideal vehicle for delivery of sulforaphane.

Methods and analysis The objective of the study is to assess the safety, pharmacokinetics and efficacy of SFX-01. This is a prospective, multicentre, randomised, double-blind placebo-controlled trial in patients aged 18-80 years with aneurysmal subarachnoid haemorrhage in the previous 48 hours. 90 patients will be randomised to receive SFX-01 $(300 \mathrm{mg})$ or placebo two times per day for up to 28 days.

Safety will be assessed using blood tests and adverse event reporting.

Pharmacokinetics will be assessed based on paired blood and cerebrospinal fluid (CSF) sulforaphane levels on day 7. A subgroup will have hourly samples taken during 6 hours post-dosing on days 1 and 7 . Pharmacodynamics will be assessed by haptoglobin and malondialdehyde levels, and maximum flow velocity of middle cerebral artery will be measured by transcranial Doppler ultrasound.

Clinical outcomes will be assessed at days 28,90 and 180 with modified Rankin Scale, Glasgow Outcome Score, SAH Outcome Tool, Short Form-36, Brain Injury Community Rehabilitation Outcome Scales and Check List for Cognitive and Emotional consequences following stroke. MRI at 6 months including quantitative susceptibility mapping and volumetric $\mathrm{T} 1$ will measure iron deposition and cortical volume.

Safety, CSF sulforaphane concentration and middle cerebral artery flow velocity will be primary outcomes and all others secondary.

Ethics and dissemination Ethical approval was obtained from South Central Hampshire A committee. Outcomes of the trial will be submitted for publication in a peerreviewed journal.
Strengths and limitations of this study

- A strength of this study is that it tests a new class of drug not previously used after human subarachnoid haemorrhage (SAH).

- It is at low risk of bias due to placebo control and appropriate blinding.

- The study design includes multiple mechanistic outcomes to give deeper understanding of any clinical findings.

- The study includes multiple novel outcome measures and MRI sequences. While these may provide new insights, some are exploratory due to limited experience with them in SAH to date.

- It is a multicentre trial, and its results should be generalisable to patients with high-volume SAH in neurosurgical units in the UK, although due to the complexity of its design, it has been limited to three centres to ensure high-quality outcome measurement.

Trial registration number NCT02614742.

\section{INTRODUCTION}

Spontaneous subarachnoid haemorrhage (SAH) is a devastating cerebrovascular injury with an incidence of 9.1 per 100000 population. ${ }^{1}$ It affects around 7000 patients in the UK annually. Around $85 \%$ are due to ruptured intracranial aneurysms. ${ }^{2}$ The incidence is age-related peaking at 52 years. SAH carries a high overall mortality rate of up to $67 \%,{ }^{3}$ and only half of the survivors are able to live independently. ${ }^{4}$ It therefore has a high burden on society due to the loss of productivity and resources. ${ }^{5}$

Conventionally following SAH, treatment is primarily directed to securing the aneurysm and prevent further re-bleeding. This however does nothing to ameliorate the morbidity and mortality due to the haemorrhage. The only approved treatment is 
nimodipine. ${ }^{6}$ However, its effects are small and poor outcome remains a significant problem. ${ }^{7}$ Moreover, even in survivors considered to have made a good recovery, neurocognitive deficits are common leading to extensive problems with social reintegration and functioning in the workplace. ${ }^{5}$

The mechanism of injury following SAH is multifactorial. ${ }^{8}$ Early brain injury (EBI) refers to the processes occurring within the first 72 hours which include blood-brain barrier (BBB) dysfunction, ${ }^{9}$ cerebral oedema, ${ }^{1011}$ neuronal cell death, ${ }^{12}$ altered ionic homeostasis, excitotoxicity, thrombin activation, ${ }^{13}$ vascular integrity degradation, ${ }^{14}$ oxidative stress ${ }^{15}$ and inflammation. ${ }^{16}$ However, despite the terminology, mechanisms such as oxidative stress and inflammation are not limited to this early period. They continue to worsen beyond the first 3 days, at the same time as CSF free haemoglobin $(\mathrm{Hb})$ concentration rises markedly as it is released form the clot and mechanisms to dispose of $\mathrm{Hb}$ are saturated. It is also in this delayed phase when cerebral vasospasm occurs, affecting both microvasculature $^{17}$ and microvasculature. ${ }^{18}$

Sulforaphane (SFN) is known to upregulate the nuclear factor erythroid 2-related factor 2 (Nrf2) pathway. Nrf2 is a redox-sensitive transcription factor that binds to a specific DNA site, the anti-oxidant response element, upstream of genes encoding detoxifying and anti-oxidant enzymes. ${ }^{190}$ Some of these enzymes include glutathione S-transferases (GSTs), NAD(P)H-quinone oxidoreductase 1 (NQO1) and haem oxygenase 1 (HO-1).$^{19}$ During physiological conditions Nrf2 is bound to the Kelch-like ECH associated protein 1 (KEAP1) in the cytoplasm. In response to stress such as SAH, Nrf2 is released from KEAP1 and then translocates to the nucleus leading to enhanced gene transcription. $^{21}$

Nrf2 also upregulates haptoglobin ( $\mathrm{Hp})$, an acute phase glycoprotein found in plasma, ${ }^{22}$ as well as cerebrospinal fluid (CSF) ${ }^{23} \mathrm{Hp}$ is part of an important $\mathrm{Hb}$ scavenging pathway after SAH. It binds tightly to free $\mathrm{Hb}$, and this Hb-Hp complex is taken up by CD163-positive macrophages. ${ }^{24}$ This pathway is saturated after $\mathrm{SAH}^{23}$ and upregulation of $\mathrm{Hp}$ represents a possible therapeutic avenue. ${ }^{25} \mathrm{Nrf2}$ also regulates degradation of red blood cells, and metabolism of haem and iron through transcriptional upregulation of CD $36,{ }^{26}$ haemopexin, ${ }^{27}$ HO- $1^{28}$ and ferritin. ${ }^{29}$

$\mathrm{Nrf2}$ is expressed in the central nervous system (CNS) and is upregulated in response to inflammation and cerebral insults. ${ }^{30}$ Nrf2 knockout is associated with a more pronounced inflammatory response in vitro ${ }^{31}$ and in vivo, ${ }^{32}$ and the increased inflammatory response is associated with more brain oedema, cell death and poorer neurological recovery. ${ }^{33} \mathrm{SFN}$ increases HO-1, NQO1 and GST- $\alpha 1$ levels and reduces IL-1 $\beta$, IL-6, and tumour necrosis factor alpha. ${ }^{34}$ It leads to a reduction in vasospasm and improves neurological recovery. ${ }^{3235}$

SFN has a relatively short half-life rendering it impractical for clinical use. SFX-01 (Evgen Pharma) is a novel new agent comprising SFN complexed with $\alpha$-cyclodextrin which is suitable for clinical use. On ingestion, $\mathrm{SFN}$ is released from the $\alpha$-cyclodextrin and is an effective method to deliver SFN. While cyclodextrin catalyses the reaction with the intermediate that is used to create the SFN, it serves a very important purpose of creating a 'scaffold' around SFN, increasing its shelflife and half-life. In two phase I trials (NCT01948362, NCT02055716) no serious adverse events (SAEs) were reported in healthy volunteers. Here we describe the protocol for a phase II trial of SFX-01 in patients who have suffered a SAH.

\section{OBJECTIVES}

The objective of the study is to assess the safety, pharmacokinetics and efficacy of SFX-01.

\section{METHODS}

\section{Trial design}

This is a prospective, double-blind, parallel group, randomised controlled trial comparing SFX-01 (300 mg) taken orally as capsules or as a suspension via a nasogastric (NG) tube two times per day for up to 28 days versus placebo in patients with SAH within 48 hours of enrolment.

The treatment window of 48 hours was selected as the best compromise between competing factors. SFX-01 has pleiotropic actions against multiple mechanisms each with different temporal profiles. Since SAH is an acute unpredictable event, the earliest one would be able to start treatment is soon after ictus, on admission into hospital. This would give optimal protection against EBI. Initiating treatment would still be justified up to 72 hours post-ictus, after which delayed cerebral ischaemia (DCI) occurs, such that a delay of more than 72 hours would be expected to undermine treatment efficacy. Within the initial 72 hours, earlier treatment would allow more time for Nrf2 pathway activation and expression of its transcriptome to protect against delayed events, as well as provide more opportunity for SFX-01 to act against EBI. On the other hand the earlier one stipulates treatment would start, the more patients one will exclude from the study. No animal studies have been conducted to investigate the timing of SFN administration, and even if available, extrapolation of timing from animal models has its limitations due to the much quicker clot resorption in rodents. After considering all these factors, it was decided to start SFX-01 treatment at the earliest possible opportunity after $\mathrm{SAH}$, yet still allow patients to be included if their presentation was delayed to some extent, to ensure generalisability to real clinical practice where delays in admission to tertiary centres are not uncommon. An audit of the lead study centre showed that most patients are admitted within 48 hours, leading to the adoption of SAH within 48 hours as 
the inclusion criterion, striking the best compromise between the practicality of recruitment and the need to start treatment early.

Detailed pharmacovigilance will inform the safety of SFX-01. Pharmacokinetics will be determined by measuring levels of SFN and its metabolites in CSF and blood at day 7 in all patients; a more detailed profile will be obtained in a subgroup of up to 12 patients who will have hourly blood and CSF samples for 6 hours after dosing on days 1 and 7. Pharmacodynamics will be assessed with blood $\mathrm{Hp}$ and malondialdehyde (MDA) levels, and middle cerebral artery (MCA) flow velocity as measured on transcranial Doppler (TCD) ultrasound as an estimate of large cerebral artery spasm. Longer term outcome will be assessed using validated outcome scales, at day 28 using modified Rankin Scale (mRS) ${ }^{36-38}$ Glasgow Outcome Score (GOSE) ${ }^{39} 40$ and the SAH Outcome Tool $(\mathrm{SAHOT})^{41}$ as well as at days 90 and 180 using the mRS, GOSE, SAHOT, Brain Injury Community Rehabilitation Outcome Scales (BICRO-39) ${ }^{42}$ and the Check List for Cognitive and Emotional consequences following stroke (CLCE-24) ${ }^{43}$

\section{Patient and public involvement}

The overall design of the study including planned investigations, that is, lumbar puncture (LP), MRI, blood tests, outcome questionnaires, the treatment methods and the consent procedures was discussed in a local SAH support group consisting of individuals with a previous history of SAH as well as their relatives. The meeting was led by the chief investigator (CI), a consultant neurovascular surgeon, and the neurovascular specialist nurse who are normally the main point of contact for SAH patients. The results were used to inform planning of the trial. Particular attention was given to the study LP which was felt to be justified on the grounds that the majority of patients will undergo CSF diversion for clinical reasons anyway, and evidence from a randomised controlled trial that CSF diversion in all Fisher grade 3 and 4 patients causes no harm and appears to provide short-term symptomatic benefit. ${ }^{44}$ The meeting was also beneficial in shaping and improving the patient information sheet and consent forms.

\section{Study setting}

This is a multicentre study conducted in regional neurosciences centres with specialist services to treat aneurysmal SAH. Patients will be identified at referral to the admitting neurosurgical or neurointensive care units. After informed consent, dosing and study interventions will occur in the neurosciences centre until patients are discharged to either home or their local hospitals. There are three recruiting centres: University Hospital Southampton, Royal Infirmary of Edinburgh and the Royal London Hospital. On discharge, follow-up may take place in clinic, or at visits to district general hospitals and rehabilitation centres, or patients' residences.
ELIGIBILITY CRITERIA

\section{Inclusion criteria}

- Patients with radiological evidence of spontaneous aneurysmal SAH.

- Fisher grade 3 or 4 on CT.

- Definitive treatment of aneurysm has not been ruled out.

- Previously living independently.

- In the opinion of the investigator, the delay from ictus to randomisation and initiation of trial medication will not exceed 48 hours.

- Aged 18 to 80 years.

- In the opinion of the investigator it will be possible to obtain informed consent from the patient, personal legal representative or professional legal representative within 24 hours of first dose.

\section{Exclusion criteria}

- Traumatic SAH.

- Fisher grade 1 or 2.

- SAH diagnosed on LP with no evidence of blood on CT.

- Decision not to treat aneurysm has been made.

- Plan to withdraw treatment.

- Significant kidney disease as defined as plasma creatinine $\geq 2.5 \mathrm{mg} / \mathrm{dL}(221 \mu \mathrm{mol} / \mathrm{l})$.

- Liver disease as defined as total bilirubin $\geq 2$ fold the upper limit of normal, as measured by the local laboratory.

- Females who are pregnant or lactating.

- Participants enrolled in another interventional research trial in the last 30 days.

- Patients for whom it is known, at the time of screening, that clinical follow-up will not be feasible.

- Patients unwilling to use two forms of contraception (one of which being a barrier method) for 90 days (men) or 30 days (women) after the last trial medication dose.

- Known hypersensitivity to any component of a SFN containing product including broccoli.

Recruitment will be limited to Fisher grade 3 and 4 SAH. These patients represent the majority of aneurysmal SAH. They have a higher volume of haemorrhage with a poorer outcome and more delayed neurological deficits. ${ }^{45}$ They are therefore mechanistically and clinically expected to derive greatest benefit from SFX-01. In addition to using Fisher scale, all baseline CT scans will undergo volumetric analysis of blood load, which are more objective and reliable than any versions of the Fisher scale.

Inclusion of patients with unsecured aneurysms would risk a high incidence of rebleeding in the study. Rebleeding is associated with exceedingly bad outcomes, ${ }^{46}$ masking any effect from SFX-01. However, since not all aneurysms are secured within 48 hours, there will be no requirement for the aneurysm to have been secured prior to enrolment. Instead patients in whom treatment of the aneurysm has been ruled out due to poor clinical status will be excluded. 
Although there are no known risks to kidney or liver, due to the relative inexperience with SFX-01 in humans, patients with liver or kidney problems will be excluded.

\section{INTERVENTION}

\section{Trial medication}

SFX-01 (active $300 \mathrm{mg}$ capsule) or placebo ( $\alpha$-cyclodextrin only capsule) will be taken orally or as a suspension via a NG two times per day for up to 28 days. PharmAgra manufactures the active pharmaceutical ingredient (API) in North Carolina, USA. The API is then encapsulated at Quotient in Reading, UK. The API manufacture has been audited by the FDA and Evgen Pharma. SFX-01 and placebo capsules as well as their contents will be identical in appearance. They will be stored at $2-8^{\circ} \mathrm{C}$.

Animal studies in ischaemic stroke, intracerebral haemorrhage and SAH have all used $5 \mathrm{mg} / \mathrm{kg}$ dose of SFN in rodents. ${ }^{32}$ 47-49 Conversion of animal doses to humans using body surface area, as has been widely recommended ${ }^{50} 51$ yields a human dose of $50 \mathrm{mg}$ SFN. This is equivalent to $300 \mathrm{mg}$ of SFX-01 containing $46.15 \mathrm{mg}$ of SFN. In the clinical studies conducted to date, SFX-01 has been shown to be well tolerated at doses of $600 \mathrm{mg}$ one time per day and $300 \mathrm{mg}$ two times per day with no SAEs. Therefore no further dose ranging studies were performed.

\section{De-escalation from the trial regimen}

In the event of tolerability problems while the patient is in the neurosurgical centre, the investigator will assess whether simple measures to ease the effects of the adverse events (AEs) may be implemented (ie, antacid in the case of gastrointestinal irritation or anti-emetic in the event of nausea).

The investigator will also assess whether or not the AEs could be related to the trial medication and severe enough to warrant a dose frequency reduction. In the first instance the investigator may consider missing one dose. If a dose frequency reduction is warranted, from that point onwards the second dose of the day will be omitted; a dose frequency increase back to two times per day will not be permitted.

If tolerability problems continue then the investigational medication will be stopped; patients will continue in the study and complete the study visits. The staged dose frequency de-escalation (dropping to one time per day) will not be carried out after discharge from the neurosurgical centre; if tolerability problems occur after discharge, medication will be stopped; patients will continue in the study and complete the study visits in accordance with the schedule of assessments.

\section{Treatment compliance}

Compliance with treatment will be recorded during the inpatient hospital stay by healthcare professionals and/or a member of the research team. On discharge to the usual residence, responsibility for this will be transferred to the patient or their personal legal representative, aided by detailed instructions. In the event of discharge to a rehabilitation unit or patient local hospital, written instructions will be provided on discharge and verbal communication with the clinical team will ensure compliance.

All patients will be discharged with a patient diary which will be filled in and collected at day 28. Compliance will be further monitored by drug reconciliation. Patients will be asked to return the medication bottle and any residual contents at the day 28 visit. At this time any residual tablets will be counted and recorded.

\section{Concomitant treatment}

There are no known drug interactions, and participation in the trial will not alter routine treatment of SAH. Participation in other interventional research studies will not be allowed until after the last follow-up visit.

\section{OUTCOMES}

\section{Primary endpoints}

Safety

To evaluate the safety of up to 28 days of SFX-01 dosed at up to an equivalent of $92 \mathrm{mg}$ SFN per day.

Prior to the start of this study, the trial medication had only been used in healthy individuals and not in a patient population. Therefore safety will be one of the main objectives of this study. This will be evaluated through routine tests (full blood count, urea and electrolytes, coagulation screen, liver function tests and urine microscopy) at baseline, post-dose, days 7 and 28 as well as close monitoring of patients for any side-effects or AEs. AEs will be coded following the Medical Dictionary for Regulatory Activities (MedDRA) and followed up until resolution and graded for severity. Incidence of dose de-escalation or discontinuation will also be reported.

\section{Pharmacokinetic}

To detect the presence of SFN and its metabolites in CSF and blood.

Animal models have shown that SFN crosses the BBB and can therefore be detected in the brain ${ }^{52} 53$ There is some variation in the levels achieved in these studies, and it has not been studied in humans. All patients will have a paired CSF/blood sample taken at 7 days post-ictus. This will be via a LP unless the patient has an external ventricular drain (EVD) for their clinical care in which case it will be obtained from the EVD. In addition, up to 12 patients with an EVD will be asked to consent to hourly CSF and blood samples for 6 hours after dosing on days 1 and 7 . Hourly CSF sampling will be performed by trained study personnel using a bespoke sterile closed cascade of syringes so that the EVD line is accessed directly only once to reduce the risk of infection. Sample collection and processing are detailed in specific study operating procedures.

\section{Vasospasm}

To determine if a minimum of 7 days treatment with SFX-01 reduces MCA peak flow velocity following SAH. 
TCD ultrasound will be used to measure the MCA, Internal Carotid Artery (ICA) and External Carotid Artery (ECA) maximum flow velocity and the Lindegaard ratio will be calculated. TCDs will be performed on alternate daily basis including at baseline. They will be performed for at least 7 days or until no longer clinically indicated. Blood flow velocity is measured in $\mathrm{cm} / \mathrm{s}$ and is inversely related to the luminal diameter of the vessel. The greater this value the more likely the degree of narrowing or spasm in the vessel. It has a good predictive value for DCI. A recent systematic review and meta-analysis, which pooled data from 2870 patients, showed a sensitivity of $90 \%$, specificity of $71 \%$ and negative and positive predictive values of $92 \%$ and $57 \%$, respectively. ${ }^{54}$

\section{Secondary endpoints}

Pharmacodynamic

CSF and blood - haptoglobin and malondialdehyde

$\mathrm{Hp}$ represents a major $\mathrm{Hb}$ detoxification pathway and is upregulated by Nrf2. MDA is a measure of oxidative stress. Hp and MDA will be measured in blood at baseline, days 7 and 28, and in CSF on day 7. A local audit at the main study neurosurgical centre showed that approximately $1 / 3$ of patients have an EVD sited as part of their routine clinical care to treat hydrocephalus. In these patients additional samples will be obtained. This will allow investigation of the temporal profile of Hp level and oxidative stress. In addition, there will be exploratory investigations using proteomics, transcriptomics and genomics using CSF and blood samples.

\section{MRI-iron and brain volume}

All patients will have MRI 180 days after SAH. Brain volume on $\mathrm{T} 1$ sequences will be measured, since this has been shown to correlate with outcome. ${ }^{55}$ Cortical iron content will be assessed using quantitative susceptibility mapping after susceptibility weighted MR imaging, which predominantly measures siderotic iron deposits. ${ }^{56}$ Iron is a major component of $\mathrm{Hb}$, and it is unknown what effects SFX-01, SFN or increased $\mathrm{Hp}$ binding of $\mathrm{Hb}$ may have on the downstream iron pathway.

\section{Clinical outcome}

- mRS at 7 days, discharge, 28, 90 and 180 days.

- Incidence of DCI defined as a new focal deficit or reduction in GCS (by two points or more) if not explained by other causes (ie, re-bleed, hydrocephalus, seizure, meningitis, sepsis or hyponatraemia). ${ }^{57}$

- Incidence of new cerebral infarct on CT or MRI.

- Institution of hypertensive therapy for presumed DCI.

- Short Form (SF)-36 quality of life survey at 28, 90 and 180 days.

- CLCE-24 and BICRO-39 at 90 and 180 days.

- SAHOT and GOSE at 28, 90 and 180 days.

- Length of acute hospital stay.

- Discharge destination.

A number of measures of efficacy have been selected reflecting the most common stroke outcome assessment
(mRS), the most common brain injury assessment (GOSE), the most common quality of life survey (SF-36) and the only SAH specific outcome tool ${ }^{41}$ to determine the most sensitive tool and make estimates of effect size. SAHOT includes 56 items dealing with cognitive, physical and behavioural/psychological consequences of $\mathrm{SAH}$, developed in a SAH focus group by patients and experts in the field. This outcome tool has been validated and proposed as a more sensitive and responsive tool in $\mathrm{SAH}^{41}$ All these tools will be administered by research nurses or doctors trained in $\mathrm{mRS}$ and GOSE in person, or in the event this is not feasible, by phone.

A number of short-term patient outcomes related to the incidence of DCI are included a priori such as incidence of infarction as adjudicated by a blinded consultant neuroradiologist (with baseline CT and follow-up MRI), and institution of hypertensive therapy which is defined as institution of inotropes to increase blood pressure in intensive care. Several simple outcomes including length of stay and discharge destination that have been associated with outcome $\mathrm{e}^{58}$ are also included a priori in the event any patients are lost to longer term follow-up.

A summary of all the key study activities with their specific time points is outlined in table 1 .

\section{SAMPLE SIZE}

No formal sample size calculation has been carried out; the power associated with a sample size of 90 is based on the following assumptions:

- The error probability for the type I error should not exceed $5 \%$ for a one-sided test.

- The primary endpoint will be compared between treatment groups by means of a t-test.

- The mean maximum MCA flow velocity for patients treated with SFX-01 is estimated as $175 \mathrm{~cm} / \mathrm{s}$.

- The SD of maximum MCA flow velocity is $50 \mathrm{~cm} / \mathrm{s}$.

Under these assumptions 90 patients will give $80 \%$ power to detect a difference in maximum MCA velocity which is approximately half of the SD of the mean value. The SD was assumed to be approximately $30 \%$ of the mean value.

\section{RECRUITMENT}

Patients with SAH who present to the clinical centres and meet the earlier criteria will be considered for recruitment. The inclusion and exclusion criteria reflect national practice. Patients will be identified by the treating clinical team at the time of referral, admission or daily medical handover.

\section{Assignment of intervention \\ Randomisation and blinding}

Patients will be randomised in a 1:1 ratio to the active or placebo arm. Randomisation will be stratified using the most recent World Federation of Neurosurgical Societies (WFNS) grade ${ }^{59}$ prior to randomisation. Patients in 
Table 1 Schedule of assessments

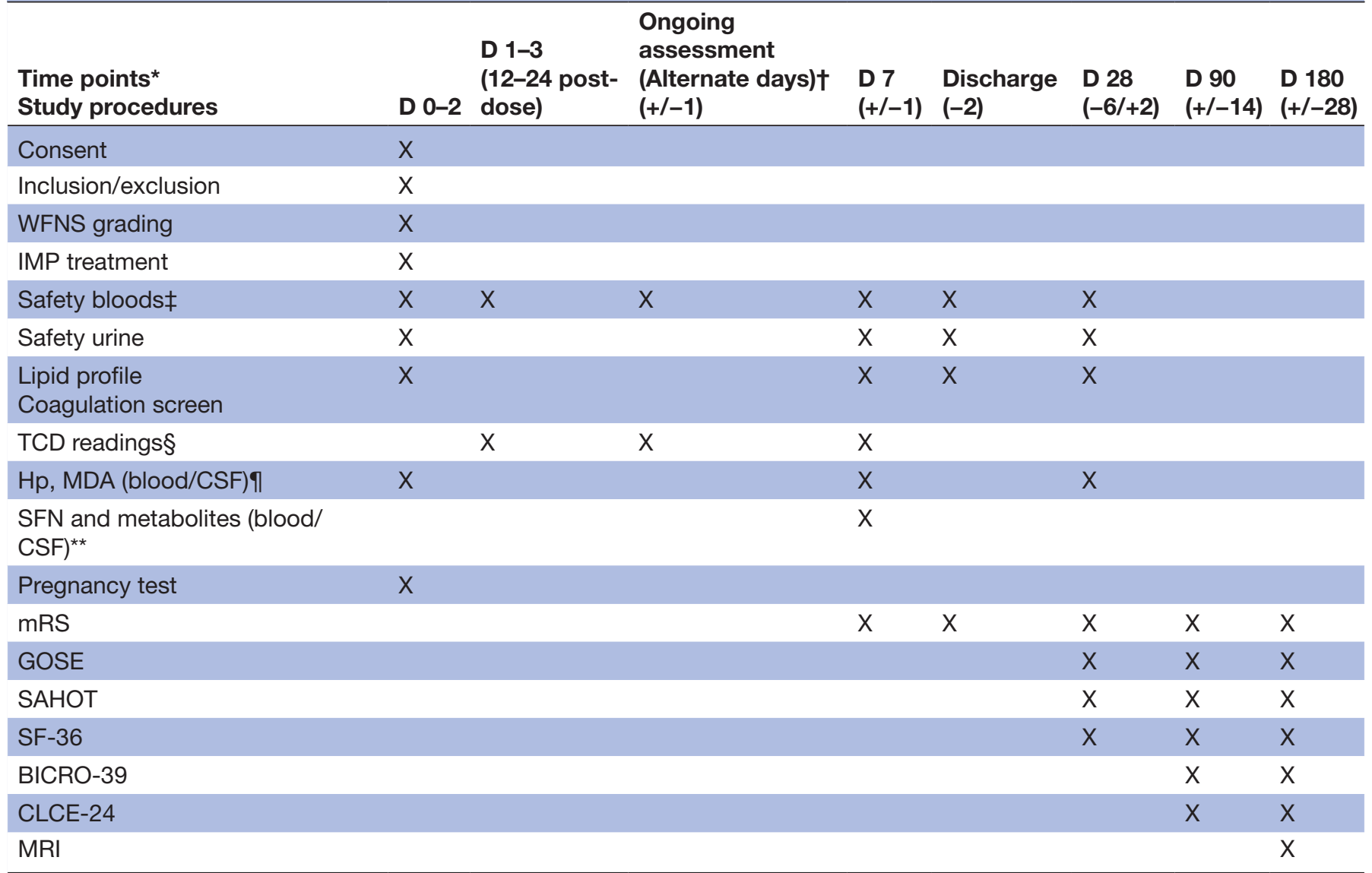

*Ictus is defined as the onset of symptoms/haemorrhage and is referred to as day 0.

$\dagger$ These assessments will be done on every other day basis with $a+/-1$ day window. They will be carried on until discharge or up to when it is clinically required.

¥Safety bloods include: biochemistry: sodium, potassium, urea, creatinine, glucose, calcium, total bilirubin, alkaline phosphatase, alanine transaminase, albumin, C-reactive protein, and haematology including haemoglobin, white blood cell count, neutrophils (absolute), lymphocytes (absolute), platelets. These will be done at least on alternate days until no longer clinically indicated.

$\S$ TCDs will be performed at baseline before day 3 and will be repeated on alternate daily basis until at least day 7 or where clinically indicated. १ Hp and MDA will be assayed in both CSF and blood at baseline, where possible, that is, if patient has an EVD fitted this will be measured in the CSF and blood at baseline as well as every other day until EVD is removed. All patients will have Hp and MDA assayed on either a LP or EVD sample on day 7.

**SFN and its metabolites will be measured on day 7 in all patient with paired blood and CSF (LP or EVD sample).

BICRO-39, Brain Injury Community Rehabilitation Outcome Scales; CLCE-24, Check List for Cognitive and Emotional consequences following stroke; CSF, cerebrospinal fluid; EVD, external ventricular drain; GOSE, Glasgow Outcome Score ; Hp, haptoglobin ; IMP, investigational medicinal product; LP, lumbar puncture; MDA, malondialdehyde; mRS, modified Rankin Scale; SAHOT, Subarachnoid Haemorrhage Outcome Tool; SF-36, Short Form-36; SFN, sulforaphane; TCD, transcranial doppler; WFNS, World Federation of Neurosurgical Societies.

different WFNS groups will have significantly different outcomes $^{60}$ and imbalance between different WFNS grades in treatment arms can introduce treatment allocation bias.

All treatment packs will be otherwise identical in appearance. Placebo capsules will be identical and contain $\alpha$-cyclodextrin making the contents indistinguishable should they be opened either inadvertently or for the purposes of NG administration. Patients will be randomised to one of the treatment groups by allocation of the appropriate, sequentially numbered treatment pack from either the clinical trials pharmacy, or a suitably calibrated and monitored fridge outside the pharmacy. The treatment packs will be pre-numbered according to a block balanced randomisation code with a ratio of $1: 1$ by a blinded third party. They will be selected as per WFNS grading by a member of a research team from pharmacy.

\section{Unblinding}

The pharmacy will receive a sealed envelope containing the identity of each trial medication bottle. An envelope may be opened only in the case of a SAE and only when it is essential to the subsequent management of the patient. The decision to unblind will be made in discussion between the treating clinician and Principal Investigator (PI) and where possible CI. The independent trial 
centre will be responsible for breaking codes for regulatory submissions of suspected unexpected serious adverse reactions (SUSARs), thereby maintaining the overall confidentiality of the code breaks. If the code is broken the data for that patient will be excluded from the per protocol population analysis but included in the intention to treat analysis. They will continue in the study and complete the study visits in accordance with the study visit schedule.

\section{DATA COLLECTION AND MANAGEMENT}

Data collection will be performed by good clinical practice-trained members of the research team. Studyspecific training and additional training in disease-specific questionnaires will be provided. The data will be entered into a secure electronic case report form.

\section{Statistical analysis}

The following populations will be considered for the analysis:

- Intention-to-treat (ITT) population: All randomised patients who receive at least one dose of study medication and with any post-dose efficacy evaluations. Patients where the time from ictus to admission is unknown are to be considered as part of the ITT population .

- Per-protocol population (PPP): The per protocol population (for primary analysis) will be considered to be those patients in the ITT population that have been dosed for a minimum to day 7 post-ictus without any major protocol violations (ie, wrong inclusions).

- Safety population: All randomised patients who have taken at least one dose of study medication.

Last observation carried forwards will be used to impute missing outcome at 6 months. The final full statistical analysis plan will be published prior to unblinding.

\section{Monitoring}

A data safety monitoring board (DSMB) has been set up to monitor the safety aspect of the trial throughout. The DSMB will be independent of the study team and company and has its own charter. A steering committee (consisting of the CI and the sponsor's chief medical officer) will receive and review the reports from DSMB, and take action as appropriate.

The first 20 patients will only be dosed as an inpatient and may therefore have courses shorter than 28 days. The DSMB plan to hold an initial meeting after recruitment of 20 patients who will have completed 7 days of trial medication. If there are no safety concerns, further patients will be allowed to be dosed with the trial drug after discharge to other hospitals or home. The DSMB will also meet if there are any SUSARs or if two patients have had an increase in the grading of the severity of AEs.

Recruitment will stop once the target has been reached or if DSMB deems the study or trial drug to be associated with a significant number of adverse events compared with the normal patient population. The recruitment target is set to a minimum of 90 patients between three centres in the UK. Up to 120 patients may be recruited in order to allow for withdrawals and deaths.

External monitoring will occur regularly throughout the study and after the study has been completed. At these visits the monitor(s) will inspect various study records, case report forms, investigator site file and source data, provided that subject confidentiality is respected.

\section{Adverse events reporting}

All AEs, adverse drug reactions and SAE will be accurately documented. The AEs are graded in both severity and seriousness, that is, mild, moderate and severe. Where severe on the severity scale subsequent SAEs will be completed instead, within 24 hours of the research team being informed. At each visit all AEs will be reassessed to ensure no change has occurred from the previous assessment. Monitoring and auditing are done against the original documentation on a 6 weekly basis by the external monitor. All AEs are also entered onto an electronic case report from where further external monitoring will be done.

Pregnancies occurring during the study must be reported immediately. In the event it does occur, patients will be referred for close obstetric monitoring. All obstetric visits will be monitored closely by the research team and any concerns will be highlighted and addressed accordingly.

\section{Data availability}

On completion of the study, data will be shared with other eligible investigators through academically established means. The data sets used and/or analysed during the study will be available from the corresponding author on reasonable request.

\section{ETHICAL CONSIDERATIONS AND INFORMED CONSENT Consent procedures and emergency dosing}

Most patients with acute SAH present with either severe headache or altered level of consciousness. Many will lack capacity with no legal representative immediately available. SAH is an acute emergency and any benefit from SFX-01 is likely to be greatest the earlier it is administered. The study has therefore been granted ethical permission to obtain baseline blood testing and administer two doses of trial drug without consent if the patient is lacking in capacity and no legal representative is available. If no consent can be obtained at that point, the patient will be withdrawn from the study.

Consent will be obtained in one of three scenarios

1. Patients with capacity.

2. Patients without capacity, but with a relative or next of kin available immediately in person. 
3. Patients without capacity and no relative or next of kin immediately available in person. In this case, a professional legal representative will be approached.

\section{Re-consent}

This must take place in two different scenarios:

1. When patients regain capacity, they must be re-consented.

2. When patients have been consented through a professional legal representative, after which time either they regain capacity or the next of kin becomes available.

\section{DISCUSSION}

There is mounting evidence supporting the role of the Nrf2 pathway in outcome after SAH. SFN upregulates Nrf2 expression and improves outcome in animal models. SFX-01 represents an exciting and novel way to deliver SFN to SAH patients with the potential to improve their lives.

This trial will investigate the safety, pharmacokinetics and pharmacodynamics of SFX-01 after SAH. If successful it may deliver long-term benefit to patients who have suffered SAH and provide new hope to a group of patients characterised by complex neurocognitive problems and disability.

\section{Twitter Ardalan H Zolnourian @Y-2383-2018}

Acknowledgements The protocol was revised and finalised in conjunction with David Howatt and Robert Holland previously at Evgen Pharma. The manuscript was reviewed by Sally Ross and Thomas Morris of Evgen Pharma.

Contributors DOB conceived the trial. AHZ, IG and DOB were all involved in the design of the study and its set-up. AHZ and DOB wrote the study protocol. DOB managed the recruitment of other centres. SF, IG and DOB reviewed the protocol manuscript and approved the final version.

Funding This study is funded and sponsored by Evgen Pharma.

Competing interests SF is the chief executive officer of Evgen Pharma plc. All the other authors have no financial or non-financial interest in Evgen Pharma plc.

Patient consent for publication Not required.

Provenance and peer review Not commissioned; externally peer reviewed.

Open access This is an open access article distributed in accordance with the Creative Commons Attribution Non Commercial (CC BY-NC 4.0) license, which permits others to distribute, remix, adapt, build upon this work non-commercially, and license their derivative works on different terms, provided the original work is properly cited, appropriate credit is given, any changes made indicated, and the use is non-commercial. See: http://creativecommons.org/licenses/by-nc/4.0/.

ORCID iD

Ardalan H Zolnourian http://orcid.org/0000-0002-0428-179X

\section{REFERENCES}

1 de Rooij NK, Linn FHH, van der Plas JA, et al. Incidence of subarachnoid haemorrhage: a systematic review with emphasis on region, age, gender and time trends. J Neurol Neurosurg Psychiatry 2007;78:1365-72.

2 van Gijn J, Rinkel GJ. Subarachnoid haemorrhage: diagnosis, causes and management. Brain 2001;124:249-78.

3 Nieuwkamp DJ, Setz LE, Algra A, et al. Changes in case fatality of aneurysmal subarachnoid haemorrhage over time, according to age, sex, and region: a meta-analysis. Lancet Neurol 2009;8:635-42.

4 Kirkpatrick P, Lindsay K, Shaw M. National study of subarachnoid haemorrhage, 2006.
5 Rivero-Arias O, Gray A, Wolstenholme J. Burden of disease and costs of aneurysmal subarachnoid haemorrhage (aSAH) in the United Kingdom. Cost Eff Resour Alloc 2010;8:6-12.

6 Pickard JD, Murray GD, Illingworth R, et al. Effect of oral nimodipine on cerebral infarction and outcome after subarachnoid haemorrhage: British aneurysm nimodipine trial. BMJ 1989;298:636-42.

7 Dorhout Mees S, Rinkel GJE, Feigin VL, et al. Calcium antagonists for aneurysmal subarachnoid haemorrhage. Cochrane Database Syst Rev 2007;308.

8 Zolnourian A, Galea I, Bulters D. Neuroprotective role of the Nrf2 pathway in subarachnoid haemorrhage and its therapeutic potential. Oxid Med Cell Longev 2019;2019:1-21.

9 Dóczi T. The pathogenetic and prognostic significance of bloodbrain barrier damage at the acute stage of aneurysmal subarachnoid haemorrhage. Clinical and experimental studies. Acta Neurochir 1985;77:110-32.

10 László FA, Varga C, Dóczi T. Cerebral oedema after subarachnoid haemorrhage. pathogenetic significance of vasopressin. Acta Neurochir 1995;133:122-33.

11 Zetterling M, Hallberg L, Ronne-Engström E. Early global brain oedema in relation to clinical admission parameters and outcome in patients with aneurysmal subarachnoid haemorrhage. Acta Neurochir 2010;152:1527-33.

12 Cahill J, Cahill WJ, Calvert JW, et al. Mechanisms of early brain injury after subarachnoid hemorrhage. J Cereb Blood Flow Metab 2006;26:1341-53.

13 Lad SP, Hegen H, Gupta G, et al. Proteomic biomarker discovery in cerebrospinal fluid for cerebral vasospasm following subarachnoid hemorrhage. J Stroke Cerebrovasc Dis 2012;21:30-41.

14 Kusaka G, Ishikawa M, Nanda A, et al. Signaling pathways for early brain injury after subarachnoid hemorrhage. J Cereb Blood Flow Metab 2004;24:916-25.

15 Gaetani P, Pasqualin A, Rodriguez y Baena R, et al. Oxidative stress in the human brain after subarachnoid hemorrhage. J Neurosurg 1998;89:748-54.

16 Fassbender K, Hodapp B, Rossol S, et al. Inflammatory cytokines in subarachnoid haemorrhage: association with abnormal blood flow velocities in basal cerebral arteries. J Neurol Neurosurg Psychiatry 2001;70:534-7.

17 Pennings FA, Bouma GJ, Ince C. Direct observation of the human cerebral microcirculation during aneurysm surgery reveals increased arteriolar contractility. Stroke 2004;35:1284-8.

18 Dorsch NW, King MT. A review of cerebral vasospasm in aneurysmal subarachnoid haemorrhage Part I: incidence and effects. J Clin Neurosci 1994;1:19-26.

19 Itoh K, Chiba T, Takahashi S, et al. An Nrf2/small Maf heterodimer mediates the induction of phase II detoxifying enzyme genes through antioxidant response elements. Biochem Biophys Res Commun 1997;236:313-22.

20 Venugopal R, Jaiswal AK. Nrf2 and Nrf1 in association with Jun proteins regulate antioxidant response element-mediated expression and coordinated induction of genes encoding detoxifying enzymes. Oncogene 1998;17:3145-56.

21 Itoh K, Ishii T, Wakabayashi N, et al. Regulatory mechanisms of cellular response to oxidative stress. Free Radic Res 1999;31:319-24.

22 Zhao X, Song S, Sun G, et al. Neuroprotective role of haptoglobin after intracerebral hemorrhage. J Neurosci 2009;29:15819-27.

23 Galea J, Cruickshank G, Teeling JL, et al. The intrathecal CD163haptoglobin-hemoglobin scavenging system in subarachnoid hemorrhage. J Neurochem 2012;121:785-92.

24 Kristiansen M, Graversen JH, Jacobsen C, et al. Identification of the haemoglobin scavenger receptor. Nature 2001;409:198-201.

25 Bulters D, Gaastra B, Zolnourian A, et al. Haemoglobin scavenging in intracranial bleeding: biology and clinical implications. Nat Rev Neurol 2018;14:416-32.

26 Zhao X, Sun G, Ting S-M, et al. Cleaning up after ICH: the role of Nrf2 in modulating microglia function and hematoma clearance. $J$ Neurochem 2015;133:144-52.

27 Morris CM, Candy JM, Edwardson JA, et al. Evidence for the localization of haemopexin immunoreactivity in neurones in the human brain. Neurosci Lett 1993;149:141-4.

28 Chen M, Regan RF. Time course of increased heme oxygenase activity and expression after experimental intracerebral hemorrhage: correlation with oxidative injury. J Neurochem 2007;103:2015-21.

29 Harada N, Kanayama M, Maruyama A, et al. Nrf2 regulates ferroportin 1-mediated iron efflux and counteracts lipopolysaccharide-induced ferroportin 1 mRNA suppression in macrophages. Arch Biochem Biophys 2011;508:101-9. 
30 Sandberg M, Patil J, D'Angelo B, et al. NRF2-regulation in brain health and disease: implication of cerebral inflammation. Neuropharmacology 2014;79:298-306.

31 Pan $\mathrm{H}$, Wang $\mathrm{H}$, Zhu L, et al. Depletion of Nrf2 enhances inflammation induced by oxyhemoglobin in cultured mice astrocytes. Neurochem Res 2011;36:2434-41.

32 Chen G, Fang Q, Zhang J, et al. Role of the Nrf2-ARE pathway in early brain injury after experimental subarachnoid hemorrhage. $J$ Neurosci Res 2011;89:515-23.

$33 \mathrm{Li} \mathrm{T}$, Wang H, Ding Y, et al. Genetic elimination of Nrf2 aggravates secondary complications except for vasospasm after experimental subarachnoid hemorrhage in mice. Brain Res 2014;1558:90-9.

34 Zhao X-D, Zhou Y-T, Lu X-J. Sulforaphane enhances the activity of the Nrf2-ARE pathway and attenuates inflammation in $\mathrm{OxyH}$-induced rat vascular smooth muscle cells. Inflamm Res 2013;62:857-63.

35 Zhao X, Wen L, Dong M, et al. Sulforaphane activates the cerebral vascular Nrf2-ARE pathway and suppresses inflammation to attenuate cerebral vasospasm in rat with subarachnoid hemorrhage. Brain Res 2016;1653:1-7.

36 Farrell B, Godwin J, Richards S, et al. The United Kingdom transient ischaemic attack (UK-TIA) aspirin trial: final results. J Neurol Neurosurg Psychiatry 1991:54:1044-54.

37 Rankin J. Cerebral vascular accidents in patients over the age of 60. III. diagnosis and treatment. Scott Med J 1957;2:255-68.

38 Quinn TJ, Dawson J, Walters MR, et al. Reliability of the modified Rankin scale: a systematic review. Stroke 2009;40:3393-5.

39 Jennett $\mathrm{B}$, Bond $\mathrm{M}$. Assessment of outcome after severe brain damage. Lancet 1975;1:480-4.

40 Jennett B, Snoek J, Bond MR, et al. Disability after severe head injury: observations on the use of the Glasgow outcome scale. $J$ Neurol Neurosurg Psychiatry 1981;44:285-93.

41 Pace A, Mitchell S, Casselden E, et al. A subarachnoid haemorrhagespecific outcome tool. Brain 2018;141:1111-21.

42 Powell JH, Beckers K, Greenwood RJ. Measuring progress and outcome in community rehabilitation after brain injury with a new assessment instrument--the BICRO-39 scales. Brain Injury Community Rehabilitation Outcome. Arch Phys Med Rehabil 1998;79:1213-25.

43 van Heugten C, Rasquin S, Winkens I, et al. Checklist for cognitive and emotional consequences following stroke (CLCE-24): development, usability and quality of the self-report version. Clin Neurol Neurosurg 2007;109:257-62.

44 Al-Tamimi YZ, Bhargava D, Feltbower RG, et al. Lumbar drainage of cerebrospinal fluid after aneurysmal subarachnoid hemorrhage: a prospective, randomized, controlled trial (LUMAS). Stroke 2012;43:677-82.

45 Fisher CM, Kistler JP, Davis JM. Relation of cerebral vasospasm to subarachnoid hemorrhage visualized by computerized tomographic scanning. Neurosurgery 1980;6:1-9.
46 Naidech AM, Janjua N, Kreiter KT, et al. Predictors and impact of aneurysm rebleeding after subarachnoid hemorrhage. Arch Neurol 2005;62:410-6.

47 Zhao J, Kobori N, Aronowski J, et al. Sulforaphane reduces infarct volume following focal cerebral ischemia in rodents. Neurosci Lett 2006;393:108-12.

48 Zhao X, Sun G, Zhang J, et al. Transcription factor Nrf2 protects the brain from damage produced by intracerebral hemorrhage. Stroke 2007;38:3280-6.

49 Alfieri A, Srivastava S, Siow RCM, et al. Sulforaphane preconditioning of the Nrf2/HO-1 defense pathway protects the cerebral vasculature against blood-brain barrier disruption and neurological deficits in stroke. Free Radic Biol Med 2013;65:1012-22.

50 Sharma V, McNeill JH. To scale or not to scale: the principles of dose extrapolation. Br J Pharmacol 2009;157:907-21.

51 Reagan-Shaw S, Nihal M, Ahmad N. Dose translation from animal to human studies revisited. Faseb J 2008;22:659-61.

52 Clarke JD, Hsu A, Williams DE, et al. Metabolism and tissue distribution of sulforaphane in Nrf2 knockout and wild-type mice. Pharm Res 2011;28:3171-9.

53 Jazwa A, Rojo Al, Innamorato NG, et al. Pharmacological targeting of the transcription factor Nrf2 at the basal ganglia provides disease modifying therapy for experimental parkinsonism. Antioxid Redox Signal 2011;14:2347-60.

54 Kumar G, Shahripour RB, Harrigan MR. Vasospasm on transcranial Doppler is predictive of delayed cerebral ischemia in aneurysmal subarachnoid hemorrhage: a systematic review and meta-analysis. $J$ Neurosurg 2016;124:1257-64.

55 Tam AKH, llodigwe D, Li Z, et al. Global cerebral atrophy after subarachnoid hemorrhage: a possible marker of acute brain injury and assessment of its impact on outcome. Acta Neurochir Suppl 2013;115:17-21.

56 Campbell N, Verschuur C, Mitchell S, et al. Hearing impairment after subarachnoid hemorrhage. Ann Clin Transl Neurol 2019;6:420-30.

57 Vergouwen MDI, Vermeulen M, van Gijn J, et al. Definition of delayed cerebral ischemia after aneurysmal subarachnoid hemorrhage as an outcome event in clinical trials and observational studies: proposal of a multidisciplinary Research Group. Stroke 2010;41:2391-5.

58 Alaraj A, Hussein AE, Esfahani DR, et al. Reducing length of stay in aneurysmal subarachnoid hemorrhage: a three year institutional experience. J Clin Neurosci 2017;42:66-70.

59 Teasdale GM, Drake CG, Hunt W, et al. A universal subarachnoid hemorrhage scale: report of a Committee of the world Federation of neurosurgical societies. J Neurol Neurosurg Psychiatry 1988;51:1457.

60 Jaja BNR, Saposnik G, Lingsma HF, et al. Development and validation of outcome prediction models for aneurysmal subarachnoid haemorrhage: the SAHIT multinational cohort study. BMJ 2018;360:j5745. 